Pacific Journal of Mathematics

MINIMUM PROBLEMS IN THE THEORY OF RIANT METRIC 


\section{MINIMUM PROBLEMS IN THE THEORY OF PSEUDO- CONFORMAL TRANSFORMATIONS AND THEIR APPLICATION TO ESTIMATION OF THE CURVATURE OF THE INVARIANT METRIC}

\section{J. M. STARK}

1. Introduction. Resume of some previous results. ${ }^{1}$ Let $B$ be a domain in the $z_{1}, z_{2}$-space ${ }^{2}$ possessing a Bergman kernel function $K^{(B)}\left(z_{1}\right.$, $\left.z_{2} ; \bar{t}_{1}, \bar{t}_{2}\right),\left(z_{1}, z_{2}\right) \in B,\left(t_{1}, t_{2}\right) \in B$. By identifying the arguments $\left(t_{1}, t_{2}\right)=\left(z_{1}, z_{2}\right)$ one obtains the function $K^{(B)} \equiv K^{(B)}\left(z_{1}, z_{2}\right) \equiv K^{(B)}\left(z_{1}, z_{2} ; \bar{z}_{1}, \bar{z}_{2}\right)$ which plays an essential role in the theory of pseudo-conformal transformations. An important application to this theory is the theorem proved by S. Bergman stating that the metric

$$
d s^{2}=\sum_{m, n=1}^{2} T_{m \bar{n}}^{(B)} d z_{m} d \bar{z}_{n}, T_{m \bar{n}}=\frac{\partial^{2} \log K^{(B)}}{\partial z_{m} \partial \bar{z}_{n}}
$$

is invariant under pseudo-conformal transformations (B. [1], p. 52). From this follows that all measures of geometric objects in $B$ which are based on the metric (1.1) are also invariant under pseudo-conformal transformations.

In the present paper we are concerned in particular with the Riemann Curvature of (1.1) in an analytic direction (see definition in section 3). Since the second derivatives of the function $\log K^{(B)}\left(z_{1}, z_{2}\right)$ are the main constituent in the definition of the curvature, we at first discuss bounds for their distortion under pseudo-conformal transformation (see Theorem 1). For this purpose, Bergman's method of the minimum integral is used (B. [3], p. 48; K. [1]; S. [1]):

Relations among various solutions of minimum problems of the type

$$
\int_{B}|f(z)|^{2} d \omega \equiv \min =\lambda_{B} \quad(d \omega=\text { volume element }),
$$

are studied (see Theorems 2 and 3). Here $f(z)$ are analytic functions,

Received September 28, 1959. The author wishes to thank Mr. M. Maschler for valueable help in preparing the manuscript for publication.

I Square brackets refer to the bibliography at the end of the paper. We use the abbreviations $\mathrm{B} .=$ Bergman, F. = Fuchs, K. = Kobayashi, S. = Stark.

2 In the present paper we consider only domains in the space of two complex variables. The generalization of the methods to the space of more complex variables involves difficulties of technical nature only. 
regular in $B$ and subject to certain auxiliary conditions. By varying these conditions, one obtains different $\lambda_{B}$ 's. (Upper and lower indices on $\lambda_{B}$ indicate the auxiliary conditions, as described, e.g., at the end of this section.) The method of the minimum integral, which is applied in order to obtain bounds and distortion theorems for various quantities having a geometrical meaning, is based on the fact that these $\lambda_{B}$ 's depend monotonically on the domain $B$ (see (1.6), (1.7)). Indeed, if, for instance, one can express these quantities and/or their distortion in terms of the $\lambda_{B}$ 's, and if one knows that there exist "domains of comparison" $I$ and $A$ such that $I \subset B \subset A$, then, using the relations among the $\lambda$ 's, one can estimate the geometrical quantities and/or their distortion in terms of the $\lambda_{I}$ 's and the $\lambda_{A}$ 's. In general, $I$ and $A$ are required to be domains for which the kernel function can be expressed in a closed form; therefore, the various $\lambda$ 's can be estimated if one knows how to express them in terms of the kernel function. This is done in B. [2], pp. 41-43, (see (1.5)), and in $\S 2$, (see (2.2)).

Using the method of the minimum integral, Fuchs [1] has obtained an expression for the curvature in analytic direction $R$, in terms of certain $\lambda_{B}$ 's, (see (3.6)). From this expression a bound for $R$ is derived in terms of the corresponding $\lambda_{I}$ 's and $\lambda_{A}$ 's, where $I$ and $A$ are domains of comparison, $I \subset B \subset A$, (see (3.7)). It is shown in Theorem 4 and in the example which follows that this bound can be sharpened if a bound for the volume of $B$ is given, and if a finite number of orthogonal functions in $B$ and certain integrals over $B$ with weighting functions depending only on $A$ are known.

In order to prove some of the relations among the various $\lambda$ 's, we use some results which were obtained in S. [1] and B. [4] p. $97 \mathrm{ff}$. These results and the definitions of the $\lambda$ 's, used in S. [1], will be stated now for the convenience of the reader:

Consider the following general minimum problem: Let $\left\{\varphi^{(\nu)}(z)\right\}, \nu=$ $1,2, \cdots$, be a system of functions orthogonal in a domain $B^{3}$ and complete for the class $\mathscr{L}^{2}(B)$. Let $\alpha_{q p}, q=1,2, \cdots, n, p=1,2, \cdots$, be a system of conplex numbers such that $\sum_{\nu=1}^{\infty}\left|\alpha_{q \nu}\right|^{2}<\infty$ for $q=1,2, \cdots, n$. Let $X_{1}, \cdots, X_{n}$ be complex numbers. Finally, let $\lambda$ represent the minimum of the integral

$$
\int_{B}|f|^{2} d \omega=\sum_{\nu=1}^{\infty} A_{\nu} \bar{A}_{\nu}, A_{\nu}=\int_{B} f \cdot \overline{\varphi^{(\nu)}} d \omega,
$$

for functions $f \in \mathscr{L}^{2}(B)$ and satisfying

$$
\sum_{\nu=m}^{\infty} A_{\nu} \alpha_{q \nu}=X_{q}, \quad q=1,2, \cdots, n ;
$$

then ([Cf. B. (2), pp. 41-43; S. (1), (2.13)])

${ }^{3}$ In the sense that $\int_{B} \varphi^{(\mu)}(z) \cdot \overline{\varphi^{\prime}(\nu)}(z) d \omega=\delta_{\mu \nu}$, where $\delta_{\mu \nu}=0$ for $\mu \neq \nu, \delta_{\nu \nu}=1 . \quad \mathscr{L}^{2}(B)$ is the class of functions $f(z)$ which are regular in $B$ and for which $\int_{B}|f(z)|^{2} d \omega<\infty$. 


$$
\lambda=-\left|\begin{array}{ll}
0 & (\bar{X})^{\prime} \\
(X) & (D)
\end{array}\right| \div|(D)|
$$

where $(X)$ is the column matrix of $n$ rows having $X_{r}$ as elements in the $r$ th row, $(\bar{X})^{\prime}$ is the transpose of $(X)$, conjugated; $(D)$ is the square matrix of $n$ rows having $\sum_{\nu=m}^{\infty} \alpha_{r \nu} \bar{\alpha}_{s \nu}$ as element in the $r$ th row, $s$ th column, and $|(D)|$ is the determinant of $(D)$.

Denote by (1)-(8) the auxiliary conditions

(1) $f(t)=1$

(2) $f(t)=0$

(3) $f_{z_{1}}(t)=1$

(4) $f_{z_{1}}(t)=0$

(5) $f_{z_{2}}(t)=1$

(6) $f_{z_{2}}(t)=0$

( 7 ) $\int_{B} f d \omega=0$

(8) $u_{1}\left(\partial f / \partial z_{1}\right)_{t}+u_{2}\left(\partial f / \partial z_{2}\right)_{t}=1, u_{1}, u_{2}$ complex numbers;

and let
(a) $\lambda_{B}^{1}$
(b) $\lambda_{B}^{01}$
(c) $\lambda_{B}^{001}$
(d) $\lambda_{B}^{* 1}$
(e) $\lambda_{B}^{* * 1}$
(f) $\lambda_{B}^{0 * 1}$
(g) $\lambda_{B}^{* 01}$
(h) $\lambda_{B}^{1 * 0}$
(i) $\lambda_{B}^{010}$
(j) $\lambda_{B}^{* 10}$
(k) $\lambda_{B}^{10}$
(1) $\lambda_{B}^{(2)}$
(m) $\lambda_{B}^{(4)}$;
$\left(\lambda_{B} \equiv \lambda_{B}(t), t \in B\right)$,

be the minima of the integral (1.2), for functions $f \in \mathscr{L}^{2}(B)$ which are normalized at $t \in B$ by the respective auxiliary conditions
(a): (1);
(b): $(2)$
(2) and
(3); (c):
(2), (4) and (5);
(d): (3);
(e): (5);
(f): (2) and (5);
$(\mathrm{g})$ : (4) and (5); (h): (1) and (6); (i): (2), (3) and (6);
$(\mathrm{j})$ : (3) and (6); (k): (1) and (4); (l): (2) and (8); (m): (8).

Let $G$ be a domain containing a domain $B, B \subset G$; we denote by
(n) $\lambda_{G B}^{1}$
(o) $\lambda_{G B}^{01}$
(p) $\lambda_{G B}^{001}$
(q) $\lambda_{G B}^{0^{*} *_{1}}$
(r) $\lambda_{G B}^{010}$
(s) $\lambda_{G B}^{(2)} ;\left(\lambda_{B} \equiv \lambda_{B}(t), t \in B\right)$,

the minima of the integral

$$
\int_{a}|f|^{2} d \omega
$$

for functions $f \in \mathscr{L}^{2}(G)$ and normalized at $t \in B$ by the conditions 

$(n):(1)$ and (7);
(o): (2), (3) and (7);
$(p):(2),(4),(5)$ and (7); (q): (2), (5) and (7);
$(\mathrm{r}):(2),(3)$
(6) and (7); (s):
$(2),(7)$ and
(8)

It follows from the definitions of the various $\lambda$ 's that

$$
\begin{aligned}
& \lambda_{I} \leq \lambda_{B} \leq \lambda_{A} \text { for domains } I, B, A \text { such that } I \subset B \subset A \\
& \lambda_{A B} \geq \lambda_{B B} \quad \text { for domains } A, B \text { such that } A \supset B
\end{aligned}
$$

(See S. [1], (3.7a). (3.7b)). From these inequalities the following result can be deduced ${ }^{4}$ :

Lemma. Let $B$ be any domain with finite Euclidian volume, in the $\left(z_{1}, z_{2}\right)$-space, such that $\operatorname{Vol} B \leq V<\infty$. Then if $I$ and $A$ are any domains $I \subset B \subset A$, we have

$$
\begin{aligned}
\left(1 / \lambda_{B}^{1}\right) & \geq\left(1 / \lambda_{A B}^{1}\right)+(1 / V) \\
\left(1 / \lambda_{B}^{01}\right) & \geq\left\{1-\left(\lambda_{I}^{1} / V\right)\right\}\left(1 / \lambda_{A B}^{01}\right)+\left(\lambda_{I}^{1} / \lambda_{A}^{* 1}\right)\left(1 / \lambda_{A}^{* 1}\right) \\
\left(1 / \lambda_{B}^{001}\right) & \geq\left\{1-\left(\lambda_{I}^{10} / V\right)\right\}\left(1 / \lambda_{A B}^{001}\right)+\left(\lambda_{I}^{10} / V\right)\left(1 / \lambda_{A}^{* 01}\right) \\
\left(1 / \lambda_{B}^{0 * 1}\right) & \geq\left\{1-\left(\lambda_{I}^{1} / V\right)\right\}\left(1 / \lambda_{A B}^{0 * 1}\right)+\left(\lambda_{I}^{1} / V\right)\left(1 / \lambda_{A}^{* * 1}\right) \\
\left(1 / \lambda_{B}^{010}\right) & \geq\left\{1-\left(\lambda_{1}^{1 * 0} / V\right)\right\}\left(1 / \lambda_{A B}^{010}\right)+\left(\lambda_{I}^{1 * 0} / V\right)\left(1 / \lambda_{A}^{* 10}\right) \\
\left(1 / \lambda_{B}^{(2)}\right) & \geq\left\{1-\left(\lambda_{I}^{1} / V\right)\right\}\left(1 / \lambda_{A B}^{(2)}\right)+\left(\lambda_{I}^{1} / V\right)\left(1 / \lambda_{A}^{(4)}\right)
\end{aligned}
$$

at $t \equiv\left(t_{1}, t_{2}\right) \in I$.

2. Distortion theorems under some assumptions about the structure of the domain. If integrals over a domain $B$ of the type

$$
\begin{aligned}
\int_{B} K^{(A)}(\zeta, \bar{t}) d \omega_{\zeta}, & \int_{B}\left(\partial K^{(A)} / \partial \bar{z}_{\nu}\right)_{(\zeta, \bar{t})} d \omega_{\zeta}, \\
\int_{B} \int_{B} K^{(A)}(\zeta, \bar{\xi}) d \omega_{\zeta} d \omega_{\xi}, & \nu=1,2,
\end{aligned}
$$

are known, where $A$ is a domain which contains the domain $B$, then in (1.8)-(1.13) the terms involving the $\lambda_{A B}$ can be evaluated. For, if $\lambda_{A B}$ is any one of the $\lambda$ 's with double subindex in (1.8)-(1.13), then the relation between $\lambda_{A B}$ and the $\lambda_{A}$ which has the same upper indices is described as follows: Let $\psi^{(\nu)}(z), z \equiv\left(z_{1}, z_{2}\right) \in A, \nu=1,2, \cdots$, be a complete orthonormal system of functions for the class $\mathscr{L}^{2}(A)$, then each function $f(z)$ of this class can be represented in the form: $f(z)=\sum_{\nu=1}^{\infty} A_{\nu} \varphi^{(\nu)}(z)$, and the series converges absolutely and uniformly in any closed subdomain of $A$. Therefore, each of the $\lambda_{A}$ 's is a special case of the general minimum problem described in (1.3), (1.4) . Thus it follows from (1.5)

4 This is Theorem 2 of (S. [1]).

${ }^{5}$ In these formulas replace $B$ by $A$. 
that each $\lambda_{A}$ can be written in the form $\lambda_{A}=-|(N)| \div|(D)|$, where $|(D)|$ is as in (1.5) and $(N)$ is the matrix whose determinant occurs in the numerator of (1.5).

Since $K^{(A)}(z, \bar{\xi})=\sum \psi^{(\nu)}(z) \overline{\psi^{(\nu)}(\xi)}$, the matrices $(N)$ and $(D)$ depend only upon $K^{(A)}(z, \bar{\xi})$ and its derivatives at the points $z=t, \bar{\xi}=\bar{t}$, and in the case of $\lambda_{A}^{(2)}$ also upon $u_{1}, u_{2}$.

Lemma. If $\lambda_{A}=-|(N)| \div|(D)|$, where $(N)$ and $(D)$ are used as explained above, then the corresponding $\lambda_{A B}$, i.e., the $\lambda_{A B}$ which has the same upper indices as $\lambda_{A}$, can be expressed in the form

$$
\lambda_{A B}=-\left|\begin{array}{cc}
(N) & (\bar{U})^{\prime} \\
(U) & E
\end{array}\right| \div\left|\begin{array}{cc}
(D) & (\bar{W})^{\prime} \\
(W) & E
\end{array}\right| .
$$

Here

$$
E \equiv \int_{B} \int_{B} K^{(A)}(\zeta, \bar{\xi}) d w_{\zeta} d w_{\xi}
$$

and

$$
\begin{aligned}
(W) & \equiv\left(W_{1}, W_{2}, \cdots, W_{n}\right), \\
(U) & \equiv\left(0, W_{1}, W_{2}, \cdots, W_{n}\right)
\end{aligned}
$$

are row matrices ${ }^{6}$, and $(\bar{U})^{\prime}$ is the transpose of the row matrix whose elements are the conjugates of the elements of $(U)$; the same rule applies to $(W)^{\prime}$. The elements $W_{\nu}$ depend only upon the expressions (2.1), and in the case of $\lambda_{A B}^{(2)}$ also upon $u_{1}, u_{2}$.

E. g., for

$$
\lambda_{A B}=\lambda_{A B}^{1}, \lambda_{A B}^{01}, \lambda_{A B}^{001}, \lambda_{A B}^{(2)},
$$

we have, using the notation

$$
K_{\nu \mu \overline{u v}}^{(A)}(\zeta, \bar{t}) \equiv\left[\partial^{\nu+\mu+u+v} K^{(A)}(\zeta, \bar{t}) / \partial \zeta_{1}^{\nu} \partial \zeta_{2}^{\mu} \partial \overline{t_{1}^{u}} \partial \overline{t_{2}^{v}}\right],
$$

$\zeta \equiv\left(\zeta_{1}, \zeta_{2}\right), \bar{t} \equiv\left(\bar{t}_{1}, \bar{t}_{2}\right)$,

$$
\begin{aligned}
& (W)=\left(\int_{B} K^{(A)}(\zeta, \bar{t}) d \omega_{\zeta}\right), \\
& (W)=\left(\int_{B} K^{(A)}(\zeta, \bar{t}) d \omega_{\zeta}, \int_{B} K_{001 \overline{0}}^{(A)}(\zeta, \bar{t}) d \omega_{\zeta}\right), \\
& (W)=\left(K^{(A)}(\zeta, \bar{t}) d \omega_{\zeta}, \int_{B} K_{001 \overline{0}}^{(A)}(\zeta, \bar{t}) d \omega_{\zeta}, \int_{B} K_{000 \overline{1}}^{(A)}(\zeta, \bar{t}) d \omega_{\zeta}\right), \\
& (W)=\left(K^{(A)}(\zeta, \bar{t}) d \omega_{\zeta}, \bar{u}_{1} \int_{B} K_{0010}^{(A)}(\zeta, \bar{t}) d \omega_{\zeta}+\bar{u}_{2} \int_{B} K_{0001}^{(A)}(\zeta, \bar{t}) d \omega_{\zeta},\right.
\end{aligned}
$$

respectively.

${ }^{6}$ Note that $(X)$ in (1.5) is a column matrix. 
Proof. We shall prove (2.2) only for the case of $\lambda_{A B}^{01}$. The proof for the other cases can be carried out along the same lines.

Let $\varphi_{\nu}(z)$ be a complete orthogonal system for the class $\mathscr{L}^{2}(A)$. Choosing $n=2, m=1, \alpha_{1 \nu}=\varphi_{\nu}(t)$,

$$
\alpha_{2 \nu}=\left.\frac{\partial \varphi_{\nu}\left(z_{1}, z_{2}\right)}{\partial z_{1}}\right|_{z=t}, \quad X_{1}=0, X_{2}=1,
$$

the general minimum problem of $\S 1$ ( $B$ is replaced by $A$ ) becomes the minimum problem for $\lambda_{A}^{01}$.

The elements of the matrix $(D)$ in (1.5) become values of the partial derivatives of the kernel function and (1.5) is reduced to

$$
\lambda_{A}^{01}=-\frac{|S|}{\left|S_{1 I}\right|}
$$

where

$$
S=\left(\begin{array}{ccc}
0 & 0 & 1 \\
0 & K_{0000}^{(A)} & K_{0010}^{(A)} \\
1 & K_{1000}^{(A)} & K_{1010}^{(\mathcal{A})}
\end{array}\right)
$$

and $S_{1 I}$ is the matrix which one obtains by deletion of the first row and the first column from the matrix $S$. Here

$$
\left.K_{\nu \mu \overline{u v}}^{(A)}=\partial^{+\mu+u+v} K^{(A)}(z, \bar{t}) / \partial z_{1}^{\nu} \partial z_{2}^{\mu} \partial \bar{t}_{1}^{u} \partial \bar{t}_{2}^{v}\right]_{\left(z_{1}, z_{2}\right)=\left(t_{1}, t_{2}\right)} .
$$

If we choose $m=1, n=3, \alpha_{1 \nu}=\varphi_{\nu}(t), \alpha_{2 \nu}=\partial \varphi_{\nu}\left(z_{1}, z_{2}\right) /\left.\partial z_{1}\right|_{z=t}$

$$
\alpha_{3 \nu}=\int_{B} \varphi_{\nu}(\zeta) d \omega_{\zeta}, X_{1}=0, X_{2}=1, X_{3}=0
$$

then the same general minimum problem becomes the minimum problem for $\lambda_{A B}^{01}$, and (1.5) becomes $\lambda_{A B}^{01}=-|T| /\left|T_{1 I}\right|$

where

$$
T=\left(\begin{array}{cccc}
0 & 0 & 1 & 0 \\
0 & K_{0000}^{(A)} & K_{0010}^{(A)} & \int_{B} K_{0000}^{(A)}(t, \bar{\xi}) d \omega_{\xi} \\
1 & K_{1000}^{(A)} & K_{10010}^{(A)} & \int_{B} K_{1000}^{(A)}(t, \bar{\xi}) d \omega_{\xi} \\
0 & \int_{B} K_{0000}^{(A)}(\zeta, \bar{t}) d \omega_{\zeta} & \int_{B} K_{0010}^{(A)}(\zeta, \bar{t}) d \omega_{\zeta} & \int_{B} \int_{B} K_{0000}^{(A)}(\zeta, \bar{\xi}) d \omega_{\zeta} d \omega_{\xi}
\end{array}\right)
$$

and $T_{1 I}$ is the matrix which one obtains by deletion of the first row and the first column from the matrix $T$. Since $K^{(A)}(z, \bar{t})=K^{(A)}(t, \bar{z})$, we have that this expression for $\lambda_{A B}^{01}$ is equivalent to (2.2). 
Other assumptions about $B$ permit further estimates: E.g., let $\left\{\varphi_{0}^{(\sigma)}\left(z_{1}, z_{2}\right)\right\}, \sigma=1,2, \cdots, r-1, \varphi_{0}^{(1)}\left(z_{1}, z_{2}\right)=$ constant, be a set of independent functions of the class $\mathscr{L}^{2}(B)$. Let $\left\{\phi^{(\sigma)}\left(z_{1}, z_{2}\right)\right\}, \sigma=1,2, \cdots, r-1$, be functions obtained by orthonormalizing over $B$ the set $\left\{\varphi_{0}^{(\sigma)}\left(z_{1}, z_{2}\right)\right\}$, $\sigma=1,2, \cdots, r-1$. If we assume that integrals over $B$ of the type

$$
\begin{aligned}
U_{n} & =\int_{B} \overline{\varphi_{0}^{(n)}(\zeta)} K^{(A)}(\zeta, \bar{t}) d \omega_{\zeta} \\
P_{m n} & =\int_{B} \int_{B} \rho_{0}^{(m)}(\zeta) \overline{\varphi_{0}^{(n)}(\xi)} K^{(A)}(\zeta, \bar{\xi}) d \omega_{\zeta} d \omega_{\xi} \\
& m, n=1,2, \cdots, r-1
\end{aligned}
$$

are known, then we can use the relation

$$
\left(1 / \lambda_{B}^{1}\right) \geq\left(1 / \lambda_{A B r}^{1}\right)+\sum_{\sigma=1}^{r-1}\left|\varphi^{(\sigma)}\right|^{2},
$$

where

$$
\lambda_{A B r}^{1}=\left|\left(P_{m n}\right)\right| \div\left|\begin{array}{ll}
K^{(A)} & \left(U_{n}\right) \\
\left(\bar{U}_{n}\right)^{\prime} & \left(P_{m n}\right)
\end{array}\right| .
$$

Here $\left(P_{m n}\right)$ is the square matrix whose elements $P_{m n}$ are given by (2.3); $\left|\left(P_{m n}\right)\right|$ is the determinant of $\left(P_{m n}\right),\left(U_{n}\right)$ is the row matrix $\left(U_{1} U_{2} \cdots U_{r-1}\right)$, and $\left(\bar{U}_{n}\right)^{\prime}$ is the transpose of $\left(U_{n}\right)$ conjugated. ${ }^{7}$ Notice that $\lambda_{A B 2}^{1}=\lambda_{A B}^{1}$. Let

$$
\begin{gathered}
\left\{\varphi_{0}^{(\sigma)}\left(z_{1}, z_{2}\right)\right\}, \quad \sigma=1,2, \cdots, r-1, \\
\left\{\alpha_{0}^{(\nu)}\left(z_{1}\right)\right\}, \nu=1,2, \cdots, p-1,\left\{\beta_{0}^{(\mu)}\left(z_{2}\right)\right\}, \mu=1,2, \cdots, q-1
\end{gathered}
$$

be sets of functions ${ }^{8}$ such that each set consists of independent functions and each function belongs to $\mathscr{L}^{2}(B)$. Further, let $\left\{\varphi^{(1)}\right\},\left\{\alpha^{(\nu)}\right\}$, $\left\{\beta^{(\nu)}\right\}, \nu=1,2, \cdots$, be sets of orthonormal functions such that each set is complete for $\mathscr{L}^{2}(B)$, and such that the first functions of each sequence are obtained respectively by orthonormalizing over $B$ the sets $\left\{\varphi_{0}^{(\sigma)}\right\}$, $\left\{\alpha_{0}^{(\nu)}\right\}$ and $\left\{\beta_{0}^{(\mu)}\right\}$. For any domain $G, G \supset B$, we define $\lambda_{G B * * q}^{01}, \lambda_{G B r}^{* 1}, \lambda_{G B * p}^{0 * 1}$, $\lambda_{G B r}^{* * 1}$, in the same manner as we defined $\lambda_{G B}^{01}, \lambda_{G B}^{* 1}, \lambda_{G B}^{0 * 1}, \lambda_{G B}^{* * 1}$, except that auxiliary condition (7) of $\S 1$ is replaced respectively by the conditions

$$
\left\{\begin{array}{l}
\int_{B} \overline{\beta_{0}^{(\mu)}} f d \omega=0, \mu=1,2, \cdots, q-1 ; \\
\int_{B} \overline{\rho_{0}^{(\sigma)}} f d \omega=0, \sigma=1,2, \cdots, r-1 ; \\
\int_{B} \overline{\alpha_{0}^{(\nu)}} f d \omega=0, \nu=1,2, \cdots, p-1 ; \text { and } \\
\int_{B} \overline{\beta_{0}^{(\mu)}} f d \omega=0, \mu=1,2, \cdots, q-1 .
\end{array}\right.
$$

7 A formula similar to $(2.4)$ is proved in B. [1, 4] for the case in which the domains are two-dimensional. (Extension to the case of four-dimensional domains offers no difficulty.)

${ }^{8}$ E. g., $\left\{\alpha_{0}^{(\nu)}\right\} \equiv\left\{1, z_{1}, \cdots, z_{1}^{p-2}\right\},\left\{\beta_{0}^{(\mu)}\right\} \equiv\left\{1, z_{2}, \cdots, z_{2}^{q-2}\right\},\left\{\varphi_{0}^{(\sigma)}\right\} \equiv\left\{1, z_{1}, z_{2}, \cdots, z_{1}^{a} \cdot z_{2}^{b}\right\}$. 
REMARK. Conditions (2.6) do not change if one replaces $\beta_{0}^{(\mu)}, \varphi_{0}^{(\sigma)}, \alpha_{0}^{(\nu)}$, by other linearly independent functions which are respectively linear combinations of the previous functions. In particular, these functions can be replaced by $\beta^{(\mu)}, \varphi^{(\sigma)}, \alpha^{(\nu)}$, respectively.

THEOREM 1. Let $B^{*}$ be the image of a domain $B$ under a pseudoconformal transformation $z_{k}^{*}=z_{k}^{*}\left(z_{1}, z_{2}\right), k=1,2$, normalized at $t$ by $\left(\partial z_{k}^{*} / \partial z_{l}\right)_{t}=\delta_{k l}$. Here $t \equiv\left(t_{1}, t_{2}\right) \in B, z_{k}^{*}\left(t_{1}, t_{2}\right) \equiv t_{k}^{*}, \delta_{\nu \nu}=1$, and $\delta_{\nu \mu}=0$ for $\nu \neq \mu$. Further, let $I$ and $A$ be domains of comparison for $B$ such that $t \in I \subset B \subset A$. Finally, let $\left\{\varphi^{(\sigma)}\left(z_{1}, z_{2}\right)\right\}, \sigma=1,2, \cdots, r-1,\left\{\alpha^{(\nu)}\left(z_{1}\right)\right\}, \nu=$ $1,2, \cdots, p-1$, and $\left\{\beta^{(\mu)}\left(z_{2}\right)\right\}, \mu=1,2, \cdots, q-1$, be three sets of functions possessing the properties

$$
\int_{B} \varphi^{(\sigma)} \overline{\varphi^{(\kappa)}} d \omega=\delta_{\sigma k}, \int_{B} \alpha^{(\nu)} \overline{\alpha^{(k)}} d \omega=\delta_{\nu k}, \int_{B} \beta^{(\mu)} \overline{\beta^{(k)}} d \omega=\delta_{\mu_{k}} .
$$

Then $^{9}$

$$
\begin{aligned}
\left\{\lambda_{I}^{1}(\right. & \left.1-\lambda_{I}^{1} \sum_{\mu=1}^{q-1}\left|\beta^{(\mu)}\right|^{2}\right)\left(1 / \lambda_{A B * * q}^{01}\right) \\
& \left.+\left(\lambda_{I}^{1}\right)^{2} \sum_{\mu=1}^{q-1}\left|\beta^{(\mu)}\right|^{2}\left[\left(1 / \lambda_{A B r}^{* 1}\right)+\sum_{\sigma=1}^{r-1}\left|\varphi_{z_{1}}^{(\sigma)}\right|^{2}\right]\right\}_{z=t} \\
& \leq\left[\partial^{2} \log K^{\left(B^{*}\right)}\left(z^{*}, \bar{t}^{*}\right) / \partial z_{1}^{*} \partial \bar{t}_{2}^{*}\right]_{z^{*}=t^{*}} \\
& \leq\left\{\left(1 / \lambda_{I}^{01}\right)\left[\left(1 / \lambda_{A B r}^{1}\right)+\sum_{\sigma=1}^{r-1}\left|\varphi^{(\sigma)}\right|^{2}\right]\right\}_{z=t}^{10}
\end{aligned}
$$

and

$$
\begin{aligned}
\left\{\lambda_{I}^{1}(\right. & \left.1-\lambda_{I}^{1} \sum_{\nu=1}^{p-1}\left|\alpha^{(\nu)}\right|^{2}\right)\left(1-\lambda_{A B * p}^{0 * 1}\right) \\
& \left.+\left(\lambda_{I}^{1}\right)^{2} \sum_{\nu=1}^{p-1}\left|\alpha^{(\nu)}\right|^{2}\left[\left(1 / \lambda_{A B r}^{* * 1}\right)+\sum_{\sigma=1}^{r-1}\left|\varphi_{z_{2}}^{(\sigma)}\right|^{2}\right]\right\}_{z=\iota} \\
& \leq\left[\partial^{2} \log K^{\left(B^{*}\right)}\left(z^{*}, \bar{t}^{*}\right) / \partial z_{2}^{*} \partial \bar{t}_{2}^{*}\right]_{z^{*}=t^{*}} \\
& \leq\left\{\left(1 / \lambda_{I}^{0 * 1}\right)\left[\left(1 / \lambda_{A B r}^{1}\right)+\sum_{\sigma=1}^{r-1}\left|\varphi^{(\sigma)}\right|^{2}\right]\right\}_{z=t} .
\end{aligned}
$$

The $\lambda$ 's bearing multiple subscripts are functions of

$$
K^{(A)}(t, \bar{t}), K_{\nu \mu \overline{u v}}^{(A)} \equiv\left[\partial^{\nu+\mu+u+v} K^{(A)}(z, \bar{t}) / \partial z_{1}^{\nu} \partial z_{2}^{\mu} \partial \overline{t_{1}^{u}} \partial \overline{t_{2}^{v}}\right]_{z=t}
$$

$\nu+\mu+u+v=1,2$, and a finite number of integrals over $B$ with weighting functions depending only upon $A$.

REMARKS: For two given domains, the theorem gives necessary conditions in terms of various properties that one of the domains can

${ }^{9}$ We use the abbreviations $\left.z \equiv\left(z_{1}, z_{2}\right), \bar{t} \equiv \overline{\left(t_{1}\right.}, \overline{t_{2}}\right)$.

10 Concerning the symbols $\lambda_{A B r}^{1}$ see pp. 6 and 14 . 
be mapped onto the other by a transformation of the type described. The middle terms in (2.8a), (2.8b) depend only upon $B^{*}, t^{*}$.

For our proof we need certain relations between the $\lambda$ 's which we formulate in the

THEOREM 2. The following relations hold:

$$
\begin{aligned}
\left(1 / \lambda_{B}^{01}\right)= & \left(1 / \lambda_{B B * * q}^{01}\right)+\lambda_{B}^{1} \cdot \sum_{\mu=1}^{q-1}\left|\beta^{(\mu)}\right|^{2} \cdot\left\{\left(1 / \lambda_{B}^{* 1}\right)-\left(1 / \lambda_{B B * * q}^{01}\right)\right\} \\
\left(1 / \lambda_{B}^{0 * 1}\right)= & \left(1 / \lambda_{B B * p}^{0 * 1}\right)+\lambda_{B}^{1} \cdot \sum_{\nu=1}^{p-1}\left|\alpha^{(\nu)}\right|^{2} \cdot\left\{\left(1 / \lambda_{B}^{* * 1}\right)-\left(1 / \lambda_{B B * p}^{0 * 1}\right)\right\} \\
\left(1 / \lambda_{B}^{* 1}\right)= & \left(1 / \lambda_{B B r}^{* 1}\right)+\sum_{\sigma=1}^{r-1}\left|\varphi_{z_{1}}^{(\sigma)}\right|^{2} \\
\left(1 / \lambda_{B}^{* * 1}\right)= & \left(1 / \lambda_{B B r}^{* * 1}\right)+\sum_{\sigma=1}^{r-1}\left|\varphi_{z_{2}}^{(\sigma)}\right|^{2} \\
\left(1 / \lambda_{B}^{01}\right) \geq & \left(1-\lambda_{I}^{1} \cdot \sum_{\mu=1}^{q-1}\left|\beta^{(\mu)}\right|^{2}\right)\left(1 / \lambda_{A B * * q}^{01}\right) \\
& +\lambda_{I}^{1} \cdot \sum_{\mu=1}^{q-1}\left|\beta^{(\mu)}\right|^{2}\left\{\left(1 / \lambda_{A B r}^{* 1}\right)+\sum_{\sigma=1}^{r-1}\left|\varphi_{z_{1}}^{(\sigma)}\right|^{2}\right\} \\
\left(1 / \lambda_{B}^{0 * 1}\right) \geq & \left(1-\lambda_{I}^{1} \cdot \sum_{\nu=1}^{p-1}\left|\alpha^{(\nu)}\right|^{2}\right)\left(1 / \lambda_{A B * p}^{0 * 1}\right) \\
& +\lambda_{I}^{1} \cdot \sum_{\nu=1}^{p-1}\left|\alpha^{(\nu)}\right|^{2}\left\{\left(1 / \lambda_{A B r}^{* * 1}\right)+\sum_{\sigma=1}^{r-1}\left|\phi_{z_{2}}^{(\sigma)}\right|^{2}\right\}
\end{aligned}
$$

Proof. To establish (2.9) we evaluate $\lambda_{B B * * q}^{01}, \lambda_{B}^{01}, \lambda_{B}^{* 1}$, and $\lambda_{B}^{1}$ using (1.5) by taking for $\left(m, n, X_{1}, X_{2}, \alpha_{1 \nu}, \alpha_{2 \nu}\right)$ the values $\left(q, 2,0,1, \beta^{(\nu)}, \beta_{z_{1}}^{(\nu)}\right)$, $\left(1,2,0,1, \beta^{(\nu)}, \beta_{z_{1}}^{(\nu)}\right),\left(1,1,1, \ldots, \beta_{z_{1}}^{(\nu)}, \ldots\right)^{11}$ and $\left(1,1,1, \ldots, \beta^{(\nu)}, \ldots\right)$ respectively, where $\beta^{(\nu)}$ and $\beta_{z_{1}}^{(\nu)}$ are evaluated at $t=\left(t_{1}, t_{2}\right)$. Now all the $\lambda$ 's of (2.9) are expressed in terms of $\left\{\beta^{(\nu)}\right\}, \nu=1,2, \cdots$, and their derivatives at $t$, and the relation between these $\lambda$ 's is easily verified to be (2.9). Equation (2.10)-(2.12) are established in the same manner by using in (1.5) for $\lambda_{B B * p}^{0 * 1}, \lambda_{B}^{0 * 1}, \lambda_{B}^{* * 1}, \lambda_{B}^{1}, \lambda_{B B r}^{* 1}, \lambda_{B}^{* 1}, \lambda_{B B r}^{* * 1}, \lambda_{B}^{* * 1}$, values of $\left(m, n, X_{1}\right.$, $\left.X_{2}, \alpha_{1 \nu}, \alpha_{2 \nu}\right)$ respectively as follows: $\left(p, 2,0,1, \alpha^{(\nu)}, \alpha_{z_{2}}^{(\nu)}\right),\left(1,2,0,1, \alpha^{(\nu)}, \alpha_{z_{2}}^{(\nu)}\right)$, $\left(1,1,1, \ldots, \alpha_{z_{2}}^{(\nu)}, \ldots\right),\left(1,1,1, \ldots, \alpha^{(\nu)}, \ldots\right),\left(r, 1,1, \ldots, \varphi_{z_{1}}^{(\nu)}-\right),\left(1,1,1, \ldots, \varphi_{z_{1}}^{(\nu)}\right.$, $-),\left(r, 1,1,-, \varphi_{z_{2}}^{(\nu)},-\right)$, and $\left(1,1,1, \ldots, \varphi_{z_{2}}^{(\nu)},-\right)$.

From the relations $\left(1 / \lambda_{B}^{1}\right) \geq \sum_{\mu=1}^{\infty}\left|\beta^{(\mu)}\right|^{2},\left(1 / \lambda_{B}^{1}\right) \geq \sum_{\nu=1}^{\infty}\left|\alpha^{(\nu)}\right|^{2}$, it follows that the coefficients before the braces in (2.9) and (2.10) are each less than or equal to 1 . By essentially the same reasoning used to derive (1.7) (see S. [1], (3.7b)), we obtain.

$$
\lambda_{A B r} \geq \lambda_{B B r}, \lambda_{A B * p} \geq \lambda_{B B * p}, \lambda_{A B * * q} \geq \lambda_{B B * * q}, A \supset B .
$$

11 "-" means no special value is required for this quantity. 
Equations (2.9) and (2.10) are of the form ${ }^{12}$

$$
\left(1 / \lambda_{B}\right)=\left(1 / \lambda_{B B(S)}\right)+\lambda_{B}^{\infty} \cdot g \cdot\left\{\left(1 / \lambda_{B}^{(*)}\right)-\left(1 / \lambda_{B B(S)}\right)\right\},
$$

where $\lambda_{B}^{\infty} \cdot g \leq 1$, and where the auxiliary condition associated with $\lambda_{B}^{(*)}$ are among the auxiliary conditions associated with $\lambda_{B B(S)}$. Hence $\lambda_{B B(S)} \geq$ $\lambda_{B}^{(*)}$, and the brace in (2.16) is non-negative. By use of (2.15), and since

$$
\begin{gathered}
\left(1 / \lambda_{B}\right) \geq\left(1 / \lambda_{B B(S)}\right)+\lambda_{I}^{\infty} \cdot g\left\{\left(1 / \lambda_{B}^{(*)}\right)-\left(1 / \lambda_{B B(S)}\right)\right\} \\
=\left(1-\lambda_{I}^{\infty} \cdot g\right)\left(1 / \lambda_{B B(S)}\right)+\lambda_{I}^{\infty} \cdot g\left(1 / \lambda_{B}^{(*)}\right),
\end{gathered}
$$

we have

$$
\left(1 / \lambda_{B}\right) \geq\left(1-\lambda_{I}^{\infty} \cdot g\right)\left(1 / \lambda_{A B(S)}\right)+\lambda_{1}^{\infty} \cdot g\left(1 / \lambda_{A}^{(*)}\right) .
$$

Using (2.15) in (2.11) and (2.12), and substituting the resulting inequalities into (2.17), we obtain (2.13) and (2.14). This completes the proof of Theorem 2 and we begin with the proof of the Theorem 1.

Since

$$
T_{\mu \nu}^{*}=\sum_{p, b=1}^{2} T_{p q} \frac{\partial z_{p}}{\partial z_{\mu}^{*}} \frac{\partial \bar{z}_{q}}{\partial \bar{z}_{\nu}^{*}}
$$

(see (1.1)), it follows from the normalization that

$$
\begin{gathered}
T_{1 \overline{1}}^{(B)}\left(t_{1}, t_{2}\right)=T_{1 \overline{1}}^{\left(B^{*}\right)}\left(t_{1}^{*}, t_{2}^{*}\right), \\
T_{2 \overline{2}}^{(B)}\left(t_{1}, t_{2}\right)=T_{2 \overline{2}}^{\left(B^{*}\right)}\left(t_{1}^{*}, t_{2}^{*}\right) .
\end{gathered}
$$

From the relation

$$
K^{(B)}\left(z_{1}, z_{2}: \bar{z}_{1}, \bar{z}_{2}\right)=K^{\left(B^{*}\right)}\left(z_{1}^{*}, z_{2}^{*} ; \bar{z}_{1}^{*}, \bar{z}_{2}^{*}\right)\left|\frac{\partial\left(z_{1}^{*}, z_{2}^{*}\right)}{\left(z_{1}, z_{2}\right)}\right|^{2}
$$

(see B. [2]), it follows that the last two equalities are equivalent to

$$
\left(\lambda_{B}^{1} / \lambda_{B}^{01}\right)_{t}=T_{\overline{11}}^{\left(B^{*}\right)} \equiv\left[\partial^{2} \log K^{\left(B^{*}\right)}\left(z_{1}^{*}, z_{2}^{*}\right) / \partial z_{1}^{*} \partial \bar{z}_{1}^{*}\right]_{\left(t_{1}^{*} \cdot t_{2}^{*}\right)}
$$

and

$$
\left(\lambda_{B}^{1} \lambda_{B}^{0 * 1}\right)_{t}=T_{2 \overline{2}}^{\left(B^{*}\right)} \equiv\left[\partial^{2} \log K^{\left(B^{*}\right)}\left(z_{1}^{*}, z_{2}^{*}\right) / \partial z_{2}^{*} \partial \bar{z}_{2}^{*}\right]_{\left(t_{1}^{*}, t_{2}^{*}\right)} .
$$

Using the bounds for $\lambda_{B}^{1}, \lambda_{B}^{01}$, and $\lambda_{B}^{0 * 1}$ as given by (2.4), (2.13), and (2.14), we obtain (2.8a) and (2.8b).

To complete the proof of Theorem 1 we need only show that in (2.8a) and $(2.8 b)$ the $\lambda$ 's bearing multiple subscripts are expressible in

$12(2.16)$ becomes $(2.9)$ if we set $\lambda_{B}=\lambda_{B}^{01}, \lambda_{B B(S)}=\lambda_{B B * * q}^{01}, \lambda_{B}^{\infty}=\lambda_{B}^{1}, \lambda_{B}^{(*)}=\lambda_{B}^{* 1}$ and $g=\sum_{\mu=1}^{q-1}\left|\beta^{(\mu)}\right|^{2} ;(2.16)$ becomes (2.10) if we set $\lambda_{B}=\lambda_{B}^{0 * 1}, \lambda_{B B(S)}=\lambda_{B B * p}^{0 * 1}, \lambda_{B}^{\infty}=\lambda_{B}^{1}, \lambda_{B}^{(*)}=$ $\lambda_{B}^{* * 1}$, and $g=\sum_{\nu=1}^{p-1}\left|\alpha^{(\nu)}\right|^{2}$. The symbol $(S)$ in $\lambda_{B B(S)}$ means that some additional conditions are superimposed in addition to the conditions $(n)-(s)$, see p. 6 . 
terms of $K^{(A)}, K_{\nu \mu \bar{u}}^{(A)}, \nu+\mu+u+v=1,2$, and a finite number of integrals over $B$ with certain weighting functions independent of $B$. That this is true for $\lambda_{A B r}^{1}$ is shown by (2.4). Let $\left\{\psi^{(\nu)}\right\}, \nu=1,2, \cdots$, be a system of orthonormal functions over $A$ complete for $\mathscr{L}^{2}(A)$. $\lambda_{A B r}^{1}$ is the minimum of

$$
\sum_{\nu=1}^{\infty} A_{\nu} \bar{A}_{\nu}, A_{\nu}=\int_{A} f \overline{\psi^{(\nu)}} d \omega,
$$

for functions $f \in \mathscr{L}^{2}(A)$ and satisfying

$$
\begin{aligned}
f_{z_{1}}(t) & =\sum_{\nu=1}^{\infty} A_{\nu} \psi_{z_{1}}^{(\nu)}(t)=1, \int_{B} \overline{\phi_{0}^{(\mu)}}(\zeta) \cdot f(\zeta) d \omega_{\zeta} \\
& =\sum_{\nu=1}^{\infty} A_{\nu} \int_{B} \overline{\rho_{0}^{(\mu)}}(\zeta) \cdot \psi^{(\nu)}(\zeta) d \omega_{\zeta}=0 \quad \mu=1,2, \cdots, r-1 .
\end{aligned}
$$

Thus $\lambda_{A B r}^{* 1}$ can be evaluated using (1.5) and taking $m=X_{1}=1, n=r$, $X_{2}=\cdots=X_{r}=0, \alpha_{1 \nu}=\psi_{z_{1}}^{(\nu)}, \alpha_{k \nu}=\int_{B} \overline{\phi_{0}^{(k-1)}(\zeta)} \cdot \psi^{(\nu)}(\zeta) d \omega_{\zeta}, 2 \leq k \leq r$. Likewise $\lambda_{A B r}^{* * 1}, \lambda_{A B * * q}^{01}$ and $\lambda_{A B * p}^{0 * 1}$ are evaluated in the form required by the theorem by substituting in (1.5) the values

$$
\begin{aligned}
m & =1, n=r, X_{1}=1, X_{2}=\cdots=X_{r}=0 \\
\alpha_{1 \nu} & =\psi_{2_{2}}^{(\nu)}, \alpha_{k \nu}=\int_{B} \overline{\rho_{0}^{(k-1)}}(\zeta) \psi^{(\nu)}(\zeta) d \omega_{\zeta}, 2 \leq k \leq r ; \\
m & =1, n=q+1, X_{1}=0, X_{2}=1, X_{3}=\cdots \\
& =X_{q+1}=0, \alpha_{1 \nu}=\psi^{(\nu)}, \alpha_{2 \nu}=\psi_{z_{1}}^{(\nu)}, \alpha_{k \nu} \\
& =\int_{B} \overline{\beta_{0}^{(k-2)}\left(\zeta_{2}\right)} \psi^{(\nu)}(\zeta) d \omega_{\zeta}, 3 \leq k \leq q+1 ; \\
m & =1, n=p+1, X_{1}=0, X_{2}=1, X_{3}=\cdots \\
& =X_{p+1}=0, \alpha_{1 \nu}=\psi^{(\nu)}, \alpha_{2 \nu} \psi_{z_{2}}^{(\nu)}, \alpha_{k \nu} \\
& =\int_{B} \overline{\alpha_{0}^{(k-2)}\left(\zeta_{1}\right)} \psi^{(\nu)}(\zeta) d \omega_{\zeta}, 3 \leq k \leq p+1
\end{aligned}
$$

respectively. This completes the proof of the theorem.

3. Curvature in an analytic direction. In this paragraph we consider the Riemann curvature of Bergman's metric.

$$
d s_{B}^{2}=\sum_{m, n=1}^{2} T_{m \bar{n}}^{(B)} d z_{m} d \bar{z}_{n}, T_{m \bar{n}}^{(B)} \equiv T_{m \bar{n}}=\frac{\partial^{2} \log K^{(B)}}{\partial z_{m} \partial \bar{z}_{n}}
$$

is the metric defined in a domain $B$, where the formal operations are carried out as if $z_{1}, z_{2}, \bar{z}_{1}, \bar{z}_{2}$ were independent coordinates. The components of the fundamental tensor of the Riemannian geometry defined by (3.1) are then

$$
\begin{gathered}
g_{11}=g_{12}=g_{22}=g_{1 \overline{1}}=g_{1 \overline{2}}=g_{\overline{2} \overline{2}}=0 \\
g_{1 \overline{1}}=\frac{1}{2} T_{\overline{1} \overline{1}}, g_{1 \overline{2}}=\frac{1}{2} T_{1 \overline{2}}, g_{2 \overline{1}}=\frac{1}{2} T_{2 \overline{1}}, g_{2 \overline{2}}=\frac{1}{2} T_{2 \overline{2}},
\end{gathered}
$$


where now

$$
\sum_{\mu, \nu=1,2, \overline{1}, \overline{2}} g_{\mu \nu} d z_{\mu} d z_{\nu}=\sum_{m, n=1}^{2} T_{m \bar{n}} d z_{m} d \bar{z}_{n}^{13}
$$

Taking the usual formula for the Riemannian curvature of the metric $\sum_{\mu, \nu=1,2, \overline{1}, \overline{2}} g_{\mu \nu} d z_{\mu} d z_{\nu}$ in the plane defined by the vectors $\left\{u_{\alpha}\right\},\left\{v_{\alpha}\right\}, \alpha=$ $1,2, \overline{1}, \overline{2}\left(v_{\bar{\alpha}}=\bar{u}_{\alpha}, v_{\bar{\alpha}}=\bar{v}_{\alpha}\right)$ we obtain

$$
\frac{\sum R_{n \mu \nu k} u_{n} v_{\mu} u_{\nu} v_{k}}{\sum\left(g_{n \nu} g_{\mu_{k}}-g_{n k} g_{\mu \nu}\right) u_{n} v_{\mu} u_{\nu} v_{k}}
$$

where

$$
\sum \equiv \sum_{n, \mu, \nu, k=1,2, \overline{1}, \overline{2}}
$$

and $R_{n \mu \nu k}$ are the usual Riemann symbols of the first kind.

If $\left\{v_{\alpha}\right\}$ belongs to the same analytic plane as $\left\{u_{\alpha}\right\}$ (i.e., if $v_{\alpha}=\alpha u_{\alpha}$, $v_{\bar{\alpha}}=\bar{a} u_{\bar{\alpha}}$, then (3.3) becomes what is called the curvature in the analytic direction $\left\{u_{\alpha}\right\}, \alpha=1,2(\mathrm{~B} .[2]$, p. 54)

$$
R=\frac{\sum R_{\bar{n} \mu \nu \bar{k}} \bar{u}_{n} u_{\mu} u_{\nu} \bar{u}_{k}}{\sum T_{\bar{n} \mu} T_{\nu \bar{k}} \bar{u}_{n} u_{\mu} u_{\nu} \bar{u}_{k}}
$$

where

$$
\begin{aligned}
\sum & \equiv \sum_{n, \mu, \nu, k=1}^{2}, R_{\bar{\lambda} \alpha \mu \bar{\beta}} \frac{\partial^{2} T_{\mu \bar{\lambda}}}{\partial z_{\alpha} \partial \bar{z}_{\beta}}+\sum_{\rho, k=1}^{2} T^{k \bar{\rho}} \cdot \frac{\partial T_{\mu_{\bar{\rho}}}}{\partial z_{\alpha}} \cdot \frac{\partial T_{k \bar{\lambda}}}{\partial \bar{z}_{\beta}} \\
T^{1 \overline{1}} & =T_{2 \bar{l}} / D, T^{1 \overline{2}}=-T_{2 \overline{1}} / D, T^{2 \overline{1}}=-T_{1 \overline{2}} / D, T^{2 \overline{2}}=T_{\overline{1}} / D, \\
D & =T_{1 \overline{1}} T_{2 \overline{2}}-T_{1 \overline{2}} \cdot T_{2 \overline{1}} .
\end{aligned}
$$

Using Bergman's method of the minimum integral, Fuchs [1] has obtained the following result. Let $\lambda_{B}^{[3]}=\lambda_{B}^{[3]}(t), t=\left(t_{1}, t_{2}\right) \in B$ denote the minimum of the integral

$$
\int_{B}|f|^{2} d \omega, d \omega=d x_{1} d y_{1} d x_{2} d y_{2}
$$

for functions $f \in \mathscr{L}^{2}(B), f \equiv f(z) \equiv f\left(z_{1}, z_{2}\right), z_{k}=x_{k}+i y_{k}, k=1,2$, and normalized by the auxiliary conditions

$$
f(t)=f_{10}(t)=f_{01}(t)=0, u_{1}^{2} f_{20}(t)+2 u_{1} u_{2} f_{11}(t)+u_{2}^{2} f_{02}(t)=1
$$

where $f_{m n}(t) \equiv\left[\partial^{m+n} f \mid \partial z_{1}^{m} \partial z_{2}^{n}\right]_{t} ; u_{1}$ and $u_{2}$ are arbitrary fixed complex numbers, then

${ }^{13}$ I.e., in the summation, both $\mu$ and $\nu$ take the values $1,2, \overline{1}, \overline{2} ; Z_{\bar{\nu}}=\overline{Z_{\nu}}$. 


$$
\begin{aligned}
\lambda_{B}^{[3]} & =\frac{1}{K(2-R) \sum T_{m \bar{n}} u_{m} \bar{u}_{n}}, \sum \equiv \sum_{m, n=1}^{2}, \\
K & \equiv K^{(B)}(t, \bar{t}), \quad T_{m \bar{n}}^{\prime}=T_{m \bar{n}}^{(B)}(t, \bar{t}) .
\end{aligned}
$$

Using (3.5), the relation $1 / K=\lambda_{B}^{1}$ and the relation

$$
d s^{2}=\sum_{\mu, \nu=1}^{2} T_{\mu \nu}^{(B)} u_{\mu} \bar{u}_{\nu}=\lambda_{B}^{1} / \lambda_{B}^{(2)}
$$

(see B. [2], p. 53), we have

$$
2-R=\lambda_{B}^{(2)} / \lambda_{B}^{[3]}, \quad R=R(t) .
$$

Since $\lambda_{B}^{(2)}$ and $\lambda_{B}^{[3]}$ are positive, it follows from (3.6) that the curvature in an arbitrary analytic direction is less than 2. (B. [2], p. 54; F. [1]).

Let $I$ and $A, t \in I \subset B \subset A$, be domains of comparison for the given domain $B$. Then from (3.6) and the monotonicity of the $\lambda$ 's, we obtain

$$
R \leq 2-\left(\lambda_{I}^{(2)} / \lambda_{A}^{[3]}\right) .
$$

We shall show that the inequality (3.7) can be improved in certain cases if information about $B$ of the following types is given: (1) Volume $B \leq V$, where $V$ is a known number, (2) a few functions orthonormal over $B$, and (3) certain moments over $B$ with weighting functions depending only upon $I$ and $A$. We assume that

$$
\text { Vol } A>V \geq \operatorname{Vol} B, \quad I \subset B \subset A \text {. }
$$

We shall show that this information leads to an improvement in (8.7) for some cases.

Define $\lambda_{B}^{[* 3]} \equiv \lambda_{B}^{[* 3]}(t), t \in B$ to be the minimum of the integral (3.4) for functions $f \in \mathscr{L}^{2}(B)$ and normalized by the auxiliary conditions $f_{10}(t)=$ $f_{01}(t)=0, u_{1}^{2} f_{20}(t)+2 u_{1} u_{2} f_{11}(t)+u_{2}^{2} f_{02}(t)=1$, where $u_{1}$ and $u_{2}$ are arbitrary fixed complex numbers.

Let

$$
\left\{\alpha^{(\nu)}\left(z_{1}\right)\right\}, \nu=1,2, \cdots, p-1,\left\{\beta^{(\mu)}\left(z_{2}\right)\right\},
$$

$\mu=1,2, \cdots, q-1$, be sets of functions satisfying

$$
\int_{B} \alpha^{(\nu)} \cdot \overline{\alpha^{(k)}} d \omega=\delta_{\nu k}, \quad \int_{B} \beta^{(\mu)} \cdot \overline{\beta^{(k)}} d \omega=\delta_{\mu_{k}},
$$

$\delta_{\rho k}=0, \rho \neq k, \delta_{k k}=1$. We define $\lambda_{G B}^{[3]}, \lambda_{G B * p}^{* 01}, \lambda_{G B * * q}^{* 01}$ for $B \subset G$ to be the minima of the integral

$$
\int_{G}|f|^{2} d \omega
$$

for functions $f \in \mathscr{L}^{2}(G)$ and normalized by the respective sets of auxiliary conditions 
1. $f(t)=f_{10}(t)=f_{01}(t)=0, u_{1}^{2} f_{20}(t)+2 u_{1} u_{2} f_{11}(t)$

$$
+u_{2}^{2} f_{02}(t)=1, \quad \int_{B} f d \omega=0
$$

2. $f_{10}(t)=0, f_{01}(t)=1, \quad \int_{B} \bar{\alpha}^{(\nu)} \cdot f d \omega=0, \nu=1,2, \cdots, p-1$;

3. $f_{10}(t)=0, f_{01}(t)=1, \quad \int_{B} \overline{\beta^{(\mu)}} \cdot f d \omega=0, \mu=1,2, \cdots, q-1$.

THEOREM 3. The following relations hold

$$
\left(1 / \lambda_{B}^{[3]}\right)=\left(1 / \lambda_{B B}^{[3]}\right)+\left(F_{B} / \operatorname{Vol} B\right) \cdot\left\{\left(1 / \lambda_{B}^{\lfloor * 3]}\right)-\left(1 / \lambda_{B B}^{[3]}\right)\right\}
$$

where $F_{B}=\lambda_{B}^{1} \lambda_{B}^{01} \lambda_{B}^{001} / \lambda_{B}^{* 1} \lambda_{B}^{* 01}$,

$$
\begin{gathered}
\left(1 / \lambda_{B}^{* 01}\right)=\left(1 / \lambda_{B B * p}^{* 01}\right)+\lambda_{B}^{* 1}\left(\sum_{\nu=1}^{p-1}\left|\alpha_{z_{1}}^{(\nu)}\right|^{2}\right)\left\{\left(1 / \lambda_{B}^{* * 1}\right)-\left(1 / \lambda_{B B * p}^{* 01}\right)\right\} \\
\left(1 / \lambda_{B}^{* 01}\right)=\left(1 / \lambda_{B B * * q}^{* 01}\right)+\sum_{\mu=1}^{q-1}\left|\beta_{z_{2}}^{(\mu)}\right|^{2} .
\end{gathered}
$$

Proof. Let $\left\{\psi^{(\sigma)}(z)\right\}, \sigma=1,2, \cdots, \psi^{(1)}=(\operatorname{Vol} B)^{-1 / 2}$, be a set of orthonormal functions complete for $\mathscr{L}^{2}(B)$. The minima $\lambda_{B}^{[3]}, \lambda_{B}^{[* 3]}$ and $\lambda_{B B}^{[3]}$ are expressed in terms of $u_{1}, u_{2}$ and sums involving the functions $\left\{\psi^{(\sigma)}\right\}$ and their derivatives by taking in (1.5) values of [m, $n, X_{1} X_{2}, X_{3}, X_{4}, \alpha_{1 v}$, $\left.\alpha_{2 \nu}, \alpha_{3 \nu}, \alpha_{4 \nu},\right]$, respectively, as follows:

$$
\begin{aligned}
& {\left[1,4,0,0,0,1, \psi^{(\nu)}(t), \psi_{10}^{(\nu)}(t), \psi_{01}^{(\nu)}(t), H\right],} \\
& {\left[1,3,0,0,1,-, \psi_{10}^{(\nu)}(t), \psi_{01}^{(\nu)}(t), H,-\right],}
\end{aligned}
$$

and

$$
\left[2,4,0,0,0,1, \psi^{(\nu)}(t), \psi_{10}^{(\nu)}(t), \psi_{01}^{(\nu)}(t), H\right]
$$

where

$$
H=u_{1}^{2} \psi_{20}^{(\nu)}(t)+2 u_{1} u_{2} \psi_{11}^{(\nu)}(t)+u_{2}^{2} \psi_{02}^{(\nu)}(t) .
$$

The minima occurring in $F_{B}$ (see (3.11)) are expressed in terms of $u_{1}, u_{2}$ and sums involving $\left\{\psi^{(\nu)}\right\}$ and their derivatives as indicated in the proof of Theorem 1. Combining the expressions for the minima so as to eliminate $u_{1}, u_{2}$ and the sums involving $\left\{\psi^{(\sigma)}\right\}$ and their derivatives, we obtain (3.11). Relations (3.12) and (3.13) are established in a similar manner. To express $\lambda_{B B * p}^{* 01}$ and $\lambda_{B B * * q}^{* 01}$ in terms of sums involving $\left\{\psi^{(\nu)}\right\}$ and their derivatives, we take in (1.5) values of $\left[m, n, X_{1}, X_{2}, \alpha_{1 v}, \alpha_{2 v}\right]$, respectively as follows: $\left[p, 2,0,1, \psi_{10}^{(\nu)}(t), \psi_{01}^{(\nu)}(t)\right],\left[q, 2,0,1, \psi_{10}^{(\nu)}(t), \psi_{01}^{(\nu)}(t)\right]$. To find similar expressions for the other minima in (3.12) and (3.13), see the proof of Theorem 2 . 
THEOREM 4. Let $B$ be a given domain in the $\left(z_{1}, z_{2}\right)$-space having interior and exterior domains of comparison $I$ and $A$. Let the point $t=\left(t_{1}, t_{2}\right) \in I$ and $R$ be the Riemann curvature at $t$ in the analytic direction $\left(u_{1}, u_{2}\right)$, of the Bergman metric (3.1) where $K \equiv K^{(B)}(z, \bar{z})$ is the kernel function of $B$. Let $\left\{\Phi^{(\sigma)}\left(z_{1}, z_{2}\right)\right\}, \sigma=1,2, \cdots, r-1,\left\{\alpha^{(\nu)}\left(z_{1}\right)\right\}, \nu=$ $1,2, \cdots, p-1,\left\{\beta^{(\mu)}\left(z_{2}\right)\right\}, \mu=1,2, \cdots, q-1$, be three sets of functions possessing the properties

$$
\begin{aligned}
& \int_{B} \varphi^{(\sigma)} \bar{\phi}^{(k)} d \omega=\delta_{\sigma k}, \quad \int_{B} \alpha^{(\nu)} \overline{\alpha^{(k)}} d \omega=\delta_{\nu k}, \quad \int_{B} \beta^{(\mu)} \overline{\beta^{(k)}} d \omega=\delta_{\mu k}, \\
& \delta_{\rho k}=0, \rho \neq k, \delta_{k k}=1 .
\end{aligned}
$$

Then

$$
R \leq 2-\lambda_{I}^{(2)} L
$$

where

$$
\begin{aligned}
L= & \left.\max \left\{\left(1 / \lambda_{A}^{[3]}\right),\left(1 / \lambda_{A B}^{[3]}\right)+(F / V)\left[1 / \lambda_{A}^{[* 3]}\right)-\left(1 / \lambda_{A B}^{[3]}\right)\right]\right\}, \\
V \geq & \operatorname{Vol} B, F=\lambda_{I}^{1} \lambda_{I}^{01} \lambda_{I}^{001} F_{1} F_{2}, \\
F_{1}= & \max \left\{\left(1 / \lambda_{A}^{* 1}\right),\left(1 / \lambda_{A B r}^{* 1}\right)+\sum_{\sigma=1}^{r-1}\left|\varphi_{z_{2}}^{(\sigma)}\right|^{2}\right\}, \\
F_{2}= & \max \left\{\left(1 / \lambda_{A}^{* 01}\right),\left(1 / \lambda_{A B * * q}^{* 01}\right)+\sum_{\mu=1}^{q-1}\left|\beta_{z_{2}}^{(\mu)}\right|^{2},\right. \\
& \left.\left(1 / \lambda_{A B * p}^{* 01}\right)+\lambda_{I}^{* 1}\left(\sum_{\nu=1}^{p-1}\left|\alpha_{z_{1}}^{(\nu)}\right|^{2}\right)\left[\left(1 / \lambda_{A}^{* * 1}\right)-\left(1 / \lambda_{A B * p}^{* 01}\right)\right]\right\},
\end{aligned}
$$

and where the $\lambda$ 's are solutions of minimum problems depending upon the domains indicated in the subscripts, and where the $\lambda$ 's bearing multiple subscripts depend only upon the kernel function of $A$, the first few derivatives of the kernel function of $A$, a finite number of integrals over $B$ with weighting functions depending only upon $A$, and in the case of $\lambda_{A B}^{[3]}$ also upon $u_{1}, u_{2}$.

Proof. From the definitions it is clear that

$$
\lambda_{B}^{[* 3]} \leq \lambda_{B}^{[3]} \leq \lambda_{B B}^{[3]}
$$

Hence (3.11) implies

$$
F_{B} / \mathrm{Vol} B \equiv\left(\lambda_{B}^{1} \cdot \lambda_{B}^{01} \cdot \lambda_{B}^{001}\right) /\left(\lambda_{B}^{* 1} \cdot \lambda_{B}^{* 01} \cdot \operatorname{Vol} B\right) \leq 1 .
$$

Using the monotonicity of the $\lambda$ 's, (3.11)-(3.13) imply

$$
\left(1 / \lambda_{B}^{[3]}\right) \geq\left(1 / \lambda_{A B}^{[3]}\right)+\left(F_{1} / V\right)\left[\left(1 / \lambda_{A}^{[* 3]}\right)-\left(1 / \lambda_{A B}^{[3]}\right)\right] .
$$

(See details of the proof of Theorem 2 in S. [1], which is the lemma in $\S 1$ of this paper.) 
where $F_{1}$ is such that $F_{B} \geq F_{1}$,

$$
\begin{aligned}
& \left(1 / \lambda_{B}^{* 01}\right) \geq\left(1 / \lambda_{A B * p}^{* 01}\right)+\lambda_{I}^{* 1}\left(\sum_{\nu=1}^{p-1}\left|\alpha_{z_{1}}^{(\nu)}\right|^{2}\right)\left[\left(1 / \lambda_{A}^{* * 1}\right)-\left(1 / \lambda_{A B * p}^{* 01}\right)\right] \\
& \left(1 / \lambda_{B}^{* 01}\right) \geq\left(1 / \lambda_{A B * * q}^{* 01}\right)+\sum_{\mu=1}^{q-1}\left|\beta_{z_{2}}^{(\mu)}\right|^{2}
\end{aligned}
$$

From (3.6) it follows that

$$
R \leq 2-\lambda_{I}^{(2)} \cdot L_{1}
$$

where $L_{1}$ is a lower bound for $1 / \lambda_{B}^{[3]}$. Combining (3.21), (3.18), (3.19), (3.20), (2.13), and (2.14), we obtain (3.15).

To express the minima bearing multiple subscripts in terms of the quantities mentioned in the theorem, we proceed as in the proof of Theorem 3.

ExAmple. If $L=1 / \lambda_{A}^{[3]}$, then (3.15) reduces to (3.7). To show that there are cases in which (3.15) is an improvement over (3.7), we proceed as follows: Let the given domain $B$ contain the origin and have as interior an exterior domain of comparison at the origin the hyperspheres

$$
I: \sum_{k=1}^{2}\left|z_{k}-\varepsilon m\right|^{2}<m^{2}
$$

and

$$
A: \sum_{k=1}^{2}\left|z_{k}-\varepsilon M\right|^{2}<M^{2}
$$

respectively.

In constructing examples we must always take $m$ and $M$ so related that $I \subset A$. We note that $I \subset A$ if $m<M$.

Define $F_{I A}$ to be

$$
F_{1 A}=\left(\lambda_{I}^{1} \lambda_{I}^{01} \lambda_{I}^{001}\right) /\left(\lambda_{A}^{* 1} \lambda_{A}^{* 01}\right)
$$

and to facilitate computing take $u_{1}=1, u_{2}=y i, y$ real. Using formula (1.5) (see also B. [2] p. 43] and the fact that the kernel functions of $I$ and $A$ are

$$
\begin{aligned}
& \left.K^{(I)}(z, \bar{z})=2 m^{2} /\left\{\pi^{2}\left[m^{2}-\sum_{k=1}^{2}\left(z_{k}-\varepsilon m\right)\right)\left(\bar{z}_{k}-\varepsilon m\right)\right]\right\}^{3}, \\
& K^{(A)}(z, \bar{z})=2 M^{2} /\left\{\pi^{2}\left[M^{2}-\sum_{k=1}^{2}\left(z_{k}-\varepsilon M\right)\left(\bar{z}_{k}-\varepsilon M\right)\right]\right\}^{3}
\end{aligned}
$$

respectively, we compute the $\lambda$ 's in terms of $\varepsilon, m, M, y$, and obtain that $\left(F_{I A} / V\right)\left(1 / \lambda_{A}^{[3]}\right)$ and $\left(1 / \lambda_{A}^{[* 3]}\right)$ can be written in the forms

$$
\left(F_{I A} / V\right)\left(1 / \lambda_{A}^{[* 3]}\right)=\frac{1}{\rho_{I}} \frac{1}{\rho^{12}} \alpha \sum_{\nu=0}^{7} U_{\nu}(y) \cdot \varepsilon^{2 \nu},
$$




$$
\left(1 / \lambda_{A}^{[3]}\right)=\alpha \sum_{\mu=0}^{4} W_{\mu}(y) \varepsilon^{2 \mu}
$$

where

$$
\begin{aligned}
\rho_{I} & =V /\left(\frac{\pi^{2} m^{4}}{2}\right)=V /(\mathrm{Vol} I) \geq 1, \\
\alpha & =\left\{\frac{48}{\pi^{2} M^{8}\left(1-2 \varepsilon^{2}\right)^{8}}\right\}, \rho=M / m>1,
\end{aligned}
$$

and where the coefficients $U_{\nu}(y)$ and $W_{\mu}(y)$ are functions of $y$ only.

Computation gives that $U_{0}(y)=(y+1)^{2}=W_{0}(y)$, and

$$
U_{1}(y)=4\left(y^{4}-2 y^{2}-3\right)=W_{1}(y) .
$$

If we let $\eta=(1 / \sqrt{2})-\varepsilon$ we obtain

$$
\sum_{\nu=2}^{7} U_{\nu}(y) \varepsilon^{2 \nu-4}=b_{0}(y)+g_{1}(y) \eta+0\left(\eta^{2}\right)
$$

and

$$
\sum_{\mu=2}^{4} W_{\mu}(y) \varepsilon^{2 \mu-4}=b_{0}(y)+g_{2}(y) \eta+0\left(\gamma^{2}\right)
$$

where $b_{0}(y)=4\left(1-2 y^{2}-3 y^{4}\right)$ and where $g_{1}(y)>g_{2}(y)$ for $|y|$ sufficiently small.

To obtain our desired example, we first choose $y$ sufficiently near zero that $g_{1}(y)>g_{2}(y)$. Then we take $\varepsilon$ positive and near to $1 / \sqrt{2}, m$ near to $M$, and $B$ such that $I \subset B \subset A$ and such that $V /(\mathrm{Vol} \mathrm{I})$ is near 1 so that

$$
\left(F_{I A} / V\right)\left(1 / \lambda_{A}^{[* 3]}\right)>\left(1 / \lambda_{A}^{[3]}\right) .
$$

This then gives the desired example, for we have

$$
L \geq(F / V)\left(1 / \lambda_{A}^{[* 3]}\right) \geq\left(F_{I A} / V\right)\left(1 / \lambda_{A}^{[* 3]}\right)>\left(1 / \lambda_{A}^{[3]}\right),
$$

so that (3.15) gives a better bound for $R$ at the origin in the direction (1,yi), $y$ real and $|y|$ small, than does (3.7). 


\section{BIBLIOGRAPHY}

S. Bergman,

1. The method of the minimum integral and the analytic continuation of functions of two complex variables, Proc. Mat. Acad. Sci., U. S. A., 27 (1941), 328-332.

2. Sur les fonctions orthogonales de plusieurs variables complexes avec les applications à la théorie des fonctions analytiques. Mémorial des Sciences Mathématiques, 106 (1947) Paris. 3. Sur la fonction-noyau d'un domaine et ses applications dans la théorie des transformations pseudo-conformes, Mémorial des Sciences Mathématiques, 108 (1948) Paris.

4. Partial differential equations, mimeographed notes, Brown University, Providence, R. I. (1941).

B. Fuchs,

1. Uber geodätische Mannigfaltigkeiten einer bei pseudo-konformen Abbildungen invarianten Riemannschen Geometrie, Recueil Math., nouv. ser., 2 (44), 567-594.

S. Kobayashi,

1. Geometry of bounded domain, Trans. of Amer. Math. Soc., 92, (1959), $267 \mathrm{ff}$.

J. M. Stark,

1. On Distortion in Pseudo-Conformal Mapping, Pacific J. Math. 6 (1956) 565-582.

Lamar State College of Technology 


\section{PACIFIC JOURNAL OF MATHEMATICS}

\section{EDITORS}

\author{
David Gilbarg \\ Stanford University \\ Stanford, California \\ F. H. Brownell \\ University of Washington \\ Seattle 5 , Washington
}

\author{
A. L. Whiteman \\ University of Southern California \\ Los Angeles 7, California \\ L. J. PAIGE \\ University of California \\ Los Angeles 24, California
}

\section{ASSOCIATE EDITORS}

\author{
E. F. BECKENBACH \\ T. M. CHERRY \\ D. DERRY
}
E. HEWITT
A. HORN
L. NACHBIN
M. OHTSUKA
H. L. ROYDEN
M. M. SCHIFFER

E. SPANIER

E. G. STRAUS

F. WOLF

\section{SUPPORTING INSTITUTIONS}

\author{
UNIVERSITY OF BRITISH COLUMBIA \\ CALIFORNIA INSTITUTE OF TECHNOLOGY \\ UNIVERSITY OF CALIFORNIA \\ MONTANA STATE UNIVERSITY \\ UNIVERSITY OF NEVADA \\ NEW MEXICO STATE UNIVERSITY \\ OREGON STATE COLLEGE \\ UNIVERSITY OF OREGON \\ OSAKA UNIVERSITY \\ UNIVERSITY OF SOUTHERN CALIFORNIA
}

\author{
STANFORD UNIVERSITY \\ UNIVERSITY OF TOKYO \\ UNIVERSITY OF UTAH \\ WASHINGTON STATE COLLEGE \\ UNIVERSITY OF WASHINGTON

AMERICAN MATHEMATICAL SOCIETY
CALIFORNIA RESEARCH CORPORATION
HUGHES AIRCRAFT COMPANY
SPACE TECHNOLOGY LABORATORIES
NAVAL ORDNANCE TEST STATION

Mathematical papers intended for publication in the Pacific Journal of Mathematics should be typewritten (double spaced), and the author should keep a complete copy. Manuscripts may be sent to any one of the four editors. All other communications to the editors should be addressed to the managing editor, L. J. Paige at the University of California, Los Angeles 24, California. ..

50 reprints per author of each article are furnished free of charge; additional copies may be obtained at cost in multiples of 50 .

The Pacific Journal of Mathematics is published quarterly, in March, June, September, and December. The price per volume (4 numbers) is $\$ 12.00$; single issues, $\$ 3.50$. Back numbers are available. Special price to individual faculty members of supporting institutions and to individual members of the American Mathematical Society: $\$ 4.00$ per volume; single issues, $\$ 1.25$.

Subscriptions, orders for back numbers, and changes of address should be sent to Pacific Journal of Mathematics, 2120 Oxford Street, Berkeley 4, California.

Printed at Kokusai Bunken Insatsusha (International Academic Printing Co., Ltd.), No. $6_{4}$ 2-chome, Fujimi-cho, Chiyoda-ku, Tokyo, Japan.

PUBLISHED BY PACIFIC JOURNAL OF MATHEMATICS, A NON-PROFIT CORPORATION

The Supporting Institutions listed above contribute to the cost of publication of this Journăl, but they are not owners or publishers and have no responsibility for its content or policies. 


\section{Pacific Journal of Mathematics}

\section{Vol. 10, No. $3 \quad$ November, 1960}

Glen Earl Baxter, An analytic problem whose solution follows from a simple

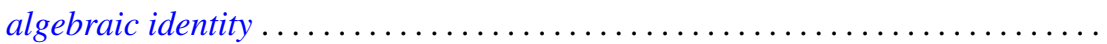

Leonard D. Berkovitz and Melvin Dresher, A multimove infinite game with linear payoff. .

Earl Robert Berkson, Sequel to a paper of A. E. Taylor ......................

Gerald Berman and Robert Jerome Silverman, Embedding of algebraic systems.... 767

Peter Crawley, Lattices whose congruences form a boolean algebra . . . . . ...... 777

Robert E. Edwards, Integral bases in inductive limit spaces . . . . . . . . . . . . . . .

Daniel T. Finkbeiner, II, Irreducible congruence relations on lattices . . . . . . . . . .

William James Firey, Isoperimetric ratios of Reuleaux polygons . . . . . . . . . . . 787

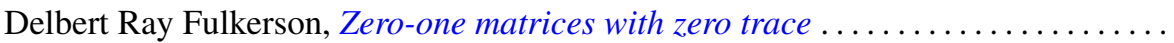

Leon W. Green, A sphere characterization related to Blaschke's conjecture........

Israel (Yitzchak) Nathan Herstein and Erwin Kleinfeld, Lie mappings in

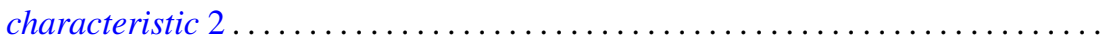

Charles Ray Hobby, A characteristic subgroup of a $p$-group .................

R. K. Juberg, On the Dirichlet problem for certain higher order parabolic

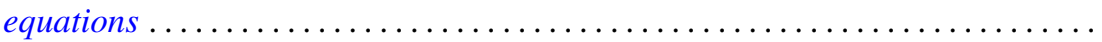

Melvin Katz, Infinitely repeatable games ......................

Emma Lehmer, On Jacobi functions . . . . . . . . . . . . . . . . . . . . . . . . .

D. H. Lehmer, Power character matrices

Henry B. Mann, A refinement of the fundamental theorem on the density of the sum of two sets of integers.

Marvin David Marcus and Roy Westwick, Linear maps on skew symmetric matrices: the invariance of elementary symmetric functions . .

Richard Dean Mayer and Richard Scott Pierce, Boolean algebras with ordered

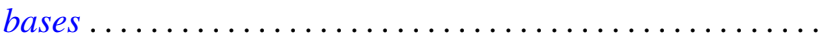

Trevor James McMinn, On the line segments of a convex surface in $E_{3} \ldots$

Frank Albert Raymond, The end point compactification of manifolds ..

Edgar Reich and S. E. Warschawski, On canonical conformal maps of regions of arbitrary connectivity

Marvin Rosenblum, The absolute continuity of Toeplitz's matrices...

Lee Albert Rubel, Maximal means and Tauberian theorems . .

Helmut Heinrich Schaefer, Some spectral properties of positive linear operators

Jeremiah Milton Stark, Minimum problems in the theory of pseudo-conformal transformations and their application to estimation of the curvature of the invariant metric.

Robert Steinberg, The simplicity of certain groups ...

Hisahiro Tamano, On paracompactness. .

Angus E. Taylor, Mittag-Leffler expansions and spectral theory .

Marion Franklin Tinsley, Permanents of cyclic matrices ...... . 